\title{
Maternal Stress Combined with Terbutaline Leads to Comorbid Autistic-Like Behavior and Epilepsy in a Rat Model
}

\author{
DFlorencia M. Bercum, ${ }^{1}$ Krista M. Rodgers, ${ }^{1}$ Alex M. Benison, ${ }^{1}$ Zachariah Z. Smith, ${ }^{1}$ Jeremy Taylor, ${ }^{1}$ Elise Kornreich, ${ }^{1}$ \\ Heidi L. Grabenstatter, ${ }^{1}$ F. Edward Dudek, ${ }^{2}$ and Daniel S. Barth ${ }^{1}$ \\ ${ }^{1}$ Department of Psychology and Neuroscience, University of Colorado, Boulder, Colorado 80309, and 2Department of Neurosurgery, University of Utah \\ School of Medicine, Salt Lake City, Utah 84108
}

Human autism is comorbid with epilepsy, yet, little is known about the causes or risk factors leading to this combined neurological syndrome. Although genetic predisposition can play a substantial role, our objective was to investigate whether maternal environmental factors alone could be sufficient.

We examined the independent and combined effects of maternal stress and terbutaline (used to arrest preterm labor), autism risk factors in humans, on measures of both autistic-like behavior and epilepsy in Sprague-Dawley rats. Pregnant dams were exposed to mild stress (foot shocks at 1 week intervals) throughout pregnancy. Pups were injected with terbutaline on postnatal days 2-5.

Either maternal stress or terbutaline resulted in autistic-like behaviors in offspring (stereotyped/repetitive behaviors and deficits in social interaction or communication), but neither resulted in epilepsy. However, their combination resulted in severe behavioral symptoms, as well as spontaneous recurrent convulsive seizures in $45 \%$ and epileptiform spikes in $100 \%$, of the rats. Hippocampal gliosis (GFAP reactivity) was correlated with both abnormal behavior and spontaneous seizures.

We conclude that prenatal insults alone can cause comorbid autism and epilepsy but it requires a combination of teratogens to achieve this; testing single teratogens independently and not examining combinatorial effects may fail to reveal key risk factors in humans. Moreover, astrogliosis may be common to both teratogens. This new animal model of combined autism and epilepsy permits the experimental investigation of both the cellular mechanisms and potential intervention strategies for this debilitating comorbid syndrome.

Key words: autism; epilepsy; maternal stress; neuroinflammation; terbutaline

Significance Statement

The comorbidity of human autism and epilepsy has been recognized for decades with little understanding of factors that increase risk. We show that two common human risk factors for autism (maternal stress and terbutaline), only when combined, result in severe ASD-like behavior and epilepsy. The significance of this work is fourfold: (1) combinations of teratogens are required to assess true risk in humans; (2) maternal stress and terbutaline, which are frequently combined in pregnant mothers, may be far more of a risk factor than previously appreciated; (3) astrogliosis may be a common mechanism for this syndrome; and (4) this first animal model of environmentally induced autism/epilepsy permits experimental investigation of cellular mechanisms and intervention strategies.

\section{Introduction}

The close association between autism spectrum disorder (ASD) and epilepsy has been recognized for decades (Tuchman et al., 2010). Approximately $30 \%$ of ASD children also have epilepsy and a similar proportion of those with epilepsy also have ASD

\footnotetext{
Received July 25, 2015; revised Oct. 19, 2015; accepted 0ct. 31, 2015.

Author contributions:F.M.B., K.M.R., and D.S.B. designed research; F.M.B., K.M.R., A.M.B., Z.Z.S., J.T., E.K., H.L.G., and D.S.B. performed research; F.M.B., K.M.R., E.K., and D.S.B. analyzed data; F.M.B., F.E.D., and D.S.B. wrote the paper.

This work was supported by the U.S. Army Medical Research and Material Command Grant PR100040 and by Autism Speaks Grant 7153.
}

(Clarke et al., 2005). Additionally, many children with ASD but without epilepsy also show subclinical (non-ictal) epileptiform abnormalities in the EEG (Ghacibeh and Fields, 2015), suggesting a prevalence of brain hyperexcitability in a larger population than

F.E.D. has equity interest in and receives consultant fees from Epitel, which is a company that makes telemetric recording devices; however, this work did not use these devices. The remaining authors declare no competing financial interests.

Correspondence should be addressed to Daniel S. Barth, Department of Psychology and Neuroscience, University of Colorado, Campus Box 345, Boulder, C0 80309. E-mail: daniel.barth@colorado.edu.

DOI:10.1523/JNEUROSCI.2803-15.2015

Copyright $\odot 2015$ the authors $\quad 0270-6474 / 15 / 3515894-09 \$ 15.00 / 0$ 
those diagnosed with convulsive seizures. The link between ASD and epilepsy suggests they may often share a common neurological basis. Although genetic predisposition can play a substantial role (Silverman et al., 2010), maternal exposure to specific environmental factors may also increase risk (Kim and Leventhal, 2015). The identification of environmental risk factors is critical because, unlike genetic risk factors, they should be preventable. A better understanding of these maternal environmental factors could therefore lead to improved strategies for decreasing risk and for intervention.

There is growing evidence that maternal stress, a risk factor in a number of human developmental disorders (Charil et al., 2010), is also linked to ASD in humans (Kinney et al., 2008). Maternal stress also results in several ASD-like behaviors (Weinstock, 2008; Patterson, 2011; typically defined as stereotyped/repetitive behaviors and deficits in social interaction and communication) and lowered thresholds for induced seizures in offspring of Sprague-Dawley rats (Edwards et al., 2002; Sadaghiani and Saboory, 2010; Qulu et al., 2012). However, maternal stress does not produce spontaneous recurrent seizures (SRSs; the hallmark of chronic epilepsy). Terbutaline, a $\beta 2$-adrenoceptor agonist commonly used to arrest preterm labor, is also considered a risk factor for autism in humans (cf. Witter et al., 2009; Rodier et al., 2011; Witter, 2011) and produces ASD-like behavior in rats (Zerrate et al., 2007), but again, it alone does not produce epilepsy.

However, given that: (1) preterm labor affects $15-18 \%$ of all pregnancies, (2) maternal stress is a known risk factor for preterm labor (Dole et al., 2003), and if it occurs between 25 and 32 weeks of gestation, (3) mothers will usually be treated with terbutaline; it is highly likely that maternal stress often precedes and accompanies terbutaline administration. This is important because both maternal stress and terbutaline have established proinflammatory effects in the CNS (Zerrate et al., 2007; Bronson and Bale, 2014) and neuroimmune inflammation has been implicated in both ASD (Vargas et al., 2005) and epilepsy (Rodgers et al., 2009; Vezzani, 2014). It is possible that their combination would deliver "two-hits" to the immune system, potentially producing behavioral and epileptogenic consequences greater than either alone.

We therefore proposed the following hypothesis: although no single prenatal environmental factor associated with ASD causes epilepsy, a combination of two factors is necessary and sufficient. To test this hypothesis, we examined the effects in rats of maternal stress combined with terbutaline on both ASD-like behavior and epileptiform activity in the EEG.

\section{Materials and Methods}

Animals and treatment. Female Sprague-Dawley rats $(n=12$, Harlan Laboratories) $220-245 \mathrm{~g}$ at embryonic day (E)2 were allowed to acclimate to the animal facility for $2 \mathrm{~d}$ before experimental manipulation. The pregnant dams were individually housed in a sealed and vented cage (Sealed Space Plus, Techniplast). Animals were housed under standard laboratory conditions in a temperature $20^{\circ} \mathrm{C} \pm 1$, and relative humidity $60 \%$ controlled environment, with a normal $12 \mathrm{~h}$ light/dark cycle (lights on 7:00 A.M.-7:00 P.M.). Food and water were available ad libitum. All procedures were performed in accordance with University of Colorado Institutional Animal Care and Use Committee guidelines for the humane use of laboratory rats in biological research.

Dams were randomly assigned to either a treatment group receiving prenatal stress $(n=5)$ or to a control nonstressed group $(n=4)$. To avoid litter size confounds due to a high mortality rate in the animals receiving both stress and terbutaline, pregnant dams were exposed to a daily mild prenatal stress paradigm from E3 through E21. On E3, each pregnant dam was placed in a sound-attenuated chamber for a $5 \mathrm{~min}$ exploration in the novel environment. To establish contextual fear conditioning, on $\mathrm{E} 4$ each dam was placed in the same environment and after an initial $60 \mathrm{~s}$ exposure, two7 $\mathrm{mA}$ shocks ( $60 \mathrm{~s}$ intershock interval) were delivered. The dam was left in the chamber for a 5 min post-shock period. Dams were then replaced in the conditioning chamber for a 5 min reexposure to the aversive environment for a subsequent $5 \mathrm{~d}$ of extinction. The paradigm was repeated weekly through out pregnancy to reinstate and maintain mild stress. Maternal weights were also recorded at E3, E9, $\mathrm{E} 15$, and E21, as well as the presence of nests in their home cage on P21 as a measure of stress. The control pregnant females were left undisturbed in their home cage.

Pups from all litters were randomized the day after birth and redistributed to the dams within their own treatment group. To ensure standardized nutrition and maternal care litter size was culled to 10 male pups per dam, allowing each dam to foster no more than two pups from their own litter. The litters were then divided into four treatment groups: controls (no maternal stress and only saline injections for the pups; "noSt $+\mathrm{Sa}$ "), those receiving terbutaline alone ("noSt $+\mathrm{TB}$ "), maternal stress alone ("St+Sa"), and those receiving the combination of maternal stress followed by terbutaline ("St+TB"). To enable comparison across all four groups, all animals were given either terbutaline sulfate (Sigma-Aldrich) or equivalent volumes of saline, administered daily on postnatal days (P)2-P5. For tocolytic therapy in humans, terbutaline doses typically lie in the range of $0.5 \mathrm{mg} / \mathrm{kg}$ per day, but can be as high as $1-2 \mathrm{mg} / \mathrm{kg}$ per day (Lam et al., 1998). In light of the fact that terbutaline has a much shorter half-life in the rat (Tegnér et al., 1984), terbutaline was administered in doses of $(10 \mathrm{mg} / \mathrm{kg})$, replicating other rat studies (Zerrate et al., 2007; Witter et al., 2009; Slotkin and Seidler, 2013) of this compound. Because the rat is an altricial species, $\mathrm{P} 2-\mathrm{P} 5$ injections have been approximated to be equivalent to the second (Rodier, 1988; Rice and Barone, 2000; Clancy et al., 2001; Zerrate et al., 2007) or third (Avishai-Eliner et al., 2002) trimester of human development, both periods of terbutaline administration where detrimental developmental consequences could be expected (Rhodes et al., 2004; Perna et al., 2014).

Repetitive behavior. P35: grooming was evaluated by placing the animal in a circular arena $(130 \mathrm{~cm}$ in diameter $) 5 \mathrm{~min}$. A blind observer scored the videos and recorded the time spent grooming for all animals.

Social exploration. Social exploration tests of anxiety (File and Seth, 2003) were conducted as described previously (Christianson et al., 2011). All animals were tested in a randomized order. At P40, treatment animals were placed in plastic tub cage $(45 \times 22 \mathrm{~cm})$ with $2 \mathrm{~cm}$ of fresh bedding located in a designated testing room for a $1 \mathrm{~h}$ habituation period. A conspecific juvenile rat was then introduced into the cage for $3 \mathrm{~min}$. The behavioral response (of the pup) to the juvenile was recorded with a video camera placed above the cage. The behavior was then assessed for each individual animal by measuring total duration and frequency of social exploration.

Ultrasonic vocalizations at P6 and P60. Ultrasonic vocalizations (USVs) were measured at P6 and P60. A wide frequency range $(15-180 \mathrm{kHz})$ microphone/preamplifier was centered $10 \mathrm{~cm}$ above a plastic container $(10 \times 10 \mathrm{~cm})$ located in a sound-attenuated chamber. USVs were digitized at $1 \mathrm{mHz}$ and analyzed for USV duration using custom-written software. At P6, each pup was taken directly from its home cage, placed in the recording chamber, and USVs recorded for $3 \mathrm{~min}$. Each pup was then weighed and placed back its home cage. At P60, each animal was placed in the recording chamber, and after a 2 min habituation period, presented with two bursts of white noise (30 ms, $110 \mathrm{db}, 2 \mathrm{~s}$ interval). USVs were recorded for $5 \mathrm{~min}$ following the last stimulus.

Composite ASD score. Because ASD-like behavior is comprised of deficits in the combined core behaviors examined here, we computed an ASD score (similar to the autism severity score put forward by El-Kordi et al., 2013) that reflected a combination of the behavioral tests. All scores across rats within a given test were first converted to $\mathrm{Z}$-scores (demeaned and divided by SD) to be of comparable magnitude between tests. Z-scores for USVs at 6 and $60 \mathrm{~d}$ as well as Z-scores for social exploration were inverted so that, when combined with grooming, greater ASD-like behavior would be consistently reflected in a higher score. Scores for the four tests were then averaged to form a composite ASD score. For graphical purposes, the composite ASD scores were scaled to a range of $0-1$ by 
subtracting the minimum value and dividing by the subsequent maximum value.

Chronic video/EEG recording. At $\sim 2$ months of age, after all behavioral data were collected and ASD-like behaviors were assessed, five rats from each group were instrumented for chronic video/EEG recording. Aseptic surgical procedures were used for all chronic preparations. Under isoflurane (Abbott Laboratories) anesthesia (2.5\%), rat pups ( 2 months of age; 275-300 g) were implanted with a Teflon-coated electrode (in an auditory responsive zone of the hippocampus; AP: $-3.8 \mathrm{~mm}, \mathrm{ML}: 1.1 \mathrm{~mm}$, DV: $3.8 \mathrm{~mm}$ ), a parietal cortical screw electrode (AP: $-2 \mathrm{~mm}$, ML: 2 $\mathrm{mm}$ ), a ground screw (AP: $-1 \mathrm{~mm}$, ML: $1 \mathrm{~mm}$ ), and a reference screw over the occipital bone. Buprenorphin $(0.1 \mathrm{mg} / \mathrm{kg}$, subcutaneously) was administered immediately after the surgical procedure and again every $12 \mathrm{~h}$ for a $72 \mathrm{~h}$ period. Following a 2 week recovery period, animals were tethered to an electrode harness (Plastics One, 363) and a slip-ring commutator (Plastics One, SL6C) permitted free movement for 24/7 video/EEG monitoring throughout the duration of the experiment. Spontaneous EEG signals were amplified and digitized at $500 \mathrm{~Hz}$ and stored in 30 min segments with time-locked video records for subsequent seizure and interictal spike analysis. Because seizures were detected in rats of the St $+\mathrm{TB}$ group in this initial experiment, four additional St $+\mathrm{TB}$ pups were prepared and recorded in a second experiment to replicate the epilepsy results.

Convulsive seizure detection and quantification. Visual detection of convulsive seizures was performed using custom software. All video/ EEG recorded 24/7 for a given rat was displayed in 30 min blocks on high-resolution monitors. All ictal-like events were examined with corresponding video to distinguish convulsive activity from potential animal-generated noise, such as eating and grooming. Verified seizures were of focal origin and secondarily generalized, and convulsive intensity was rated according to Racine's behavioral scale (Racine, 1972). Seizure onset times and behavioral intensities were digitally logged. EEG was simultaneously examined for interictal spikes distinct from convulsive seizures.

Interictal spike detection and quantification. Supervised pattern recognition was used to quantify interictal spikes (IISs), but did not replace visual examination of all video/EEG to detect seizures. In rats where IISs could be visually identified, $20-30$ were digitally marked, and then averaged to form an individualized template for each animal. This template was moved through all raw EEG data for that animal and the covariance between template and signal computed in time increments of $1 \mathrm{~ms}$. When the covariance exceeded an operator-determined threshold, the putative IIS was extracted from the raw data and added to a separate spike $\log$ for further analysis. Template matching methods such as this are subject to numerous false-positives. For this reason, we trained a support vector machine (SVM; Orrù et al., 2012) to automatically discriminate between IISs and noise (including seizures). Training was done iteratively, and at each iteration, all putative spikes (aligned to the spike waveform peak $\pm 150 \mathrm{~ms}$ ) were displayed on a high-resolution monitor and a subset of $\sim 10$ correctly and incorrectly identified events were labeled and used to initially train the SVM model. This model then classified all of the spikes, which were redisplayed with their tentative classification and another subset of correctly and incorrectly identified spikes labeled for refinement of the SVM model. Typically within 2-3 iterations, the SVM correctly accepted $>90 \%$ of the actual spikes and rejected all previously misidentified events.

Immunohistochemistry. Rats were intracardially perfused with $0.9 \%$ saline followed by $4 \%$ paraformaldehyde, tissue was collected and stored at $-80^{\circ} \mathrm{C}$. Tissue was sectioned at $20 \mu \mathrm{m}$ and mounted onto SuperFrost Plus slides (Fisher Scientific) using a cryostat. Reactive astrogliosis in the hippocampus was assessed with an astrocyte marker (glial fibrillary acidic protein; GFAP), using an avidin-biotin-horseradish peroxidase (ABC) reaction. Sections were treated with $0.3 \% \mathrm{H}_{2} \mathrm{O}_{2}$ for $30 \mathrm{~min}$ at room temperature, washed in $\mathrm{PBS}$, and incubated at $4^{\circ} \mathrm{C}$ overnight in mouse anti-GFAP (1:100; MP Biomedicals). The next day, sections were incubated at room temperature for $2 \mathrm{~h}$ with biotinylated goat anti-mouse IgG antibody (1:200; Jackson ImmunoResearch). Sections were then washed and incubated for $2 \mathrm{~h}$ at room temperature in $\mathrm{ABC}$ (1:400 Vector Laboratories) and reacted with 3', 3-diaminobenzidine (Sigma-Aldrich).
Sections were air-dried overnight and then dehydrated with graded alcohols, cleared in Histoclear and coverslipped with Permount (Fisher Scientific).

Image analysis. Digital images from the apex of dentate gyrus where the dorsal an ventral horn meet, a contralateral region homologous to the depth electrode placement, were captured with an Olympus BX-61 microscope, using Olympus Microsuite software (Olympus America), with bright-field illumination at $10 \times$ magnification. A $300 \mu \mathrm{m}^{2}$ box was placed within the region of interest, and densitometric analysis was performed, under blinded conditions, in NIH ImageJ. Signal pixels of a region-of-interest were defined as having gray values $3.5 \mathrm{SD}$ above the mean gray value of a cell-poor area close to the region of interest. The number of pixels and the average gray values above the set background were then computed for each region of interest and multiplied, giving an integrated densitometric measurement. Six measurements were made for each animal; the measurements were then averaged to obtain a single integrated density value per rat.

Statistics. Results are expressed as mean \pm SEM. A one-way repeatedmeasures ANOVA was used to compare the effect of stress (shock) on freezing behavior. A $\chi^{2}$ test of goodness-of-fit was performed to determine whether nesting behavior was expressed equally between prenatally stressed dams compared with controls and $t$ tests were used to analyze weight in dams (E3, E9, E15, and E21) and pups (P2), as well as litter size. All other analysis used a two-way ANOVA with stress and terbutaline as the independent factors. Fischer's LSD was used to conduct planned pairwise comparisons to follow-up significant overall ANOVAs. Data were analyzed using SPSS Statistics software and, in all cases, statistical significance was set at $p<0.05$.

\section{Results}

\section{Prenatal measures of stress}

Time spent freezing was significantly higher after shock compared with habituation (recorded on day E3) on all 3 shock days throughout pregnancy (main effect: $F_{(3,9)}=14.11, p=0.014$ ); pairwise comparisons: $\mathrm{E} 4(p=0.022), \mathrm{E} 11(p=0.010)$, and E18 $(p=0.044)$. Nesting behavior was found to be significantly lower in prenatally stressed dams compared with controls $\left(\chi_{(1, N=8)}^{2}\right)$, $p=0.001)$.

\section{Perinatal measures of stress}

Birth weight was significantly lower in prenatally stressed (PS) pups $(6.3 \pm 0.12 \mathrm{~g})$ compared with controls $(6.82 \pm 0.10 \mathrm{~g} ; p=$ $0.033)$. Prenatal stress had no significant effect on overall prenatal weight gain (control: $132.5 \pm 6.88$; PS: $120 \pm 8.16 \mathrm{~g}$ ) or litter size (control: $11.25 \pm 1.44$; PS: $12.25 \pm 0.55$ ).

\section{ASD-like behavior}

ANOVA indicated that there were significant differences between groups for each behavioral measure (Grooming: $F_{(3,36)}=3.27$, $p=0.032$; USV P6: $F_{(3,36)}=2.97, p=0.045$; USV P60: $F_{(3,34)}=$ 4.284, $p=0.011$; Social Exploration: $\left.F_{(3,34)}=2.91, p=0.049\right)$. However, the effects of maternal stress or terbutaline differed depending on the particular behavioral measure. The main effect of stress was significant for impairments of USVs produced by pups (P6) when separated from their dams (Fig. $1 A ; p=0.01$ ) and for repetitive behavior (Fig. $1 D ; p=0.011$ ), terbutaline was significant for social exploration (Fig. $1 C ; p=0.028$ ), and both stress and terbutaline were significant for USVs at P60 evoked by sudden sounds (Fig. $1 B ; p=0.020$ and 0.021 , respectively). Paired comparisons indicated that only the combination of maternal stress and terbutaline $(\mathrm{St}+\mathrm{TB})$ had consistent effects on all ASD-like behaviors compared with controls (noST + Sa), with significant impairments in USVs at P6 (Fig. $1 A ; p=0.008$ ) and P60 (P60; $p=0.002$; Fig. $1 B)$, as well as spending less time with social exploration (Fig. $1 C ; p=0.024$ ) and more time par- 
A

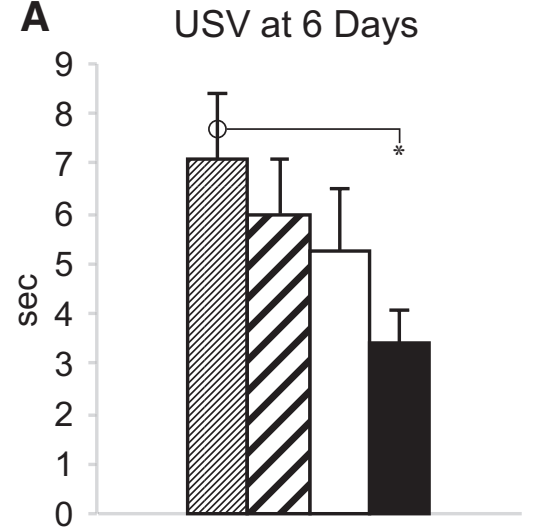

C

Social Exploration: Time Spent Sniffing

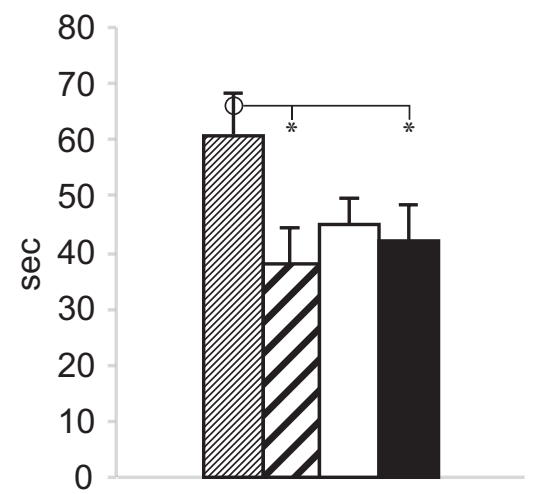

B

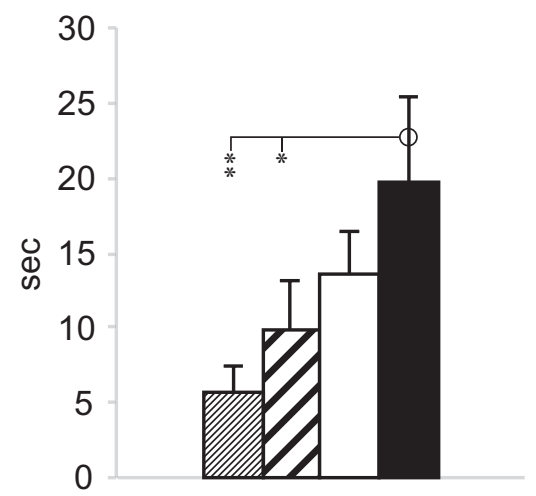

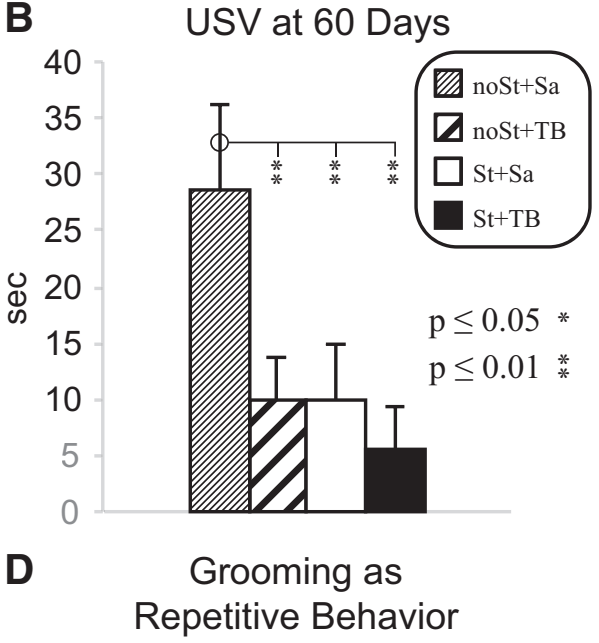

E

ASD score

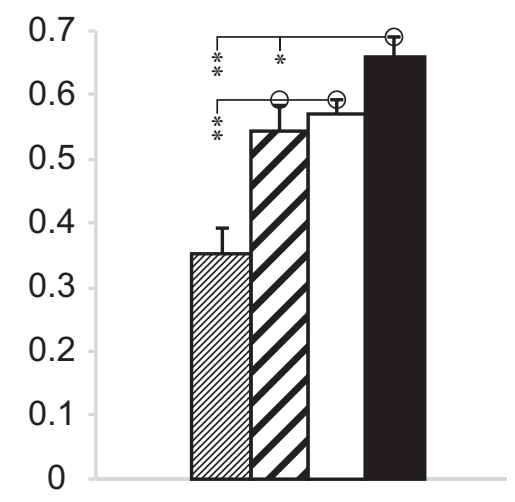

Figure 1. ASD-like behaviors. A, At P6, USVs were only significantly reduced in St + TB rats compared with noSt + Sa controls. Both noSt $+\mathrm{TB}$ and $\mathrm{St}+\mathrm{Sa}$ groups showed reduced vocalization that did not reach significance. $B$, By $\mathrm{P} 60$, all experimental animals displayed significantly reduced USVs. C, St+TB rats groomed more than noSt + Sa and noSt + TB. D, All experimental groups showed reduced social exploration. $\boldsymbol{E}$, A composite ASD score was computed by averaging the normalized values within each of the four behavioral tests, inverted for USVs at 6 and $60 \mathrm{~d}$ and for social exploration so that all had the same direction as grooming. All experimental groups (noSt $+\mathrm{TB}, \mathrm{St}+\mathrm{Sa}$, and St $+\mathrm{TB}$ ) showed significantly greater ASD-like behavior compared with controls, and noSt $+\mathrm{TB}$ was significantly less than $\mathrm{St}+\mathrm{TB}$.

ticipating in repetitive behavior (Fig. $1 D ; p=0.039$ ). Single teratogens only had effects on subsets of these behaviors compared with controls. Terbutaline alone (noSt $+\mathrm{TB})$ significantly decreased USVs at P60 $(p=0.01)$ and also decreased social exploration $(p=0.009)$. Stress alone $(\mathrm{St}+\mathrm{Sa})$ also significantly influenced USVs at P60 $(p=0.01)$ but had no effects on other behavioral measures compared with controls.
Although only the combination of terbutaline with stress was sufficient to cause significant impairments on all behavioral tests, and thus by definition, ASD, there were no significant interactions between stress and terbutaline on any particular behavior. This outcome suggested that the effect of maternal stress may have added to that of terbutaline to produce sufficient impairments to reach significance compared with controls, but that stress did not alter (ie, enhance) the effect of terbutaline. To further explore this issue, we devised a composite ASD behavior score that combined the results of all four tests, thus decreasing the overall variance and permitting more sensitive detection of treatment differences on ASD as a whole (Fig. $1 E)$. ANOVA of the ASD scores indicated significant main effects for both stress and terbutaline $\left(F_{(1,36)}=24.12\right.$ and 16.08, respectively, $p<0.001$ for both). Paired comparisons indicated that all three conditions (noSt $+\mathrm{TB}, \mathrm{St}+\mathrm{Sa}$, and $\mathrm{St}+\mathrm{TB})$ produced significantly higher ASD scores $(p<0.001)$ than controls but only noSt $+\mathrm{TB}$ was significantly lower than $\mathrm{St}+\mathrm{TB}(p=0.02)$. There was no interaction between stress and terbutaline $\left(F_{(1,36)}=2.35, p=0.13\right)$, indicating again no enhancement of ASD due to their combination.

\section{Seizures and epileptiform spikes}

Nearly one-half (4/9) of the St+TB rats were also epileptic, as determined by recording of SRSs ( $1.5 \pm 0.4$ min duration). The electrographic seizures had a classic complex-partial profile beginning with a progressive increase in spike amplitude and frequency, which later decreased in frequency (Fig. 2B) and were followed by post-ictal (post-seizure) depression for $2.1 \pm 0.5 \mathrm{~min}$ (Fig. 2C). The seizures typically began in the hippocampus and spread to the cortex (Fig. $2 B$; arrow).

Figure $3 A$ displays a raster plot of the same seizure depicted in Figure 2 but over a longer time-scale, combining the seizure, post-ictal depression, and IISs that typically increased after recovery from post-ictal depression. Detected IISs are highlighted in red. Typically within 2-3 iterations of training, spike detection correctly accepted $>90 \%$ of the actual spikes and rejected nearly all artifacts (Fig. $3 A$ ) including seizures (Fig. 3A, Seizure) and slow sleep waves (Fig. $3 B$, Sleep), whereas missing a minority of IISs with differing shape (Fig. $3 A$, ovals).

ANOVA indicated that there were significant differences between groups for both seizure and IIS rates (seizures: $F_{(3,32)}=$ $6.23, p=0.002$; IIS: $\left.F_{(3,32)}=19.14, p<0.001\right)$. Only St + TB rats had seizures and this significantly differed $(p<0.001)$ from all 


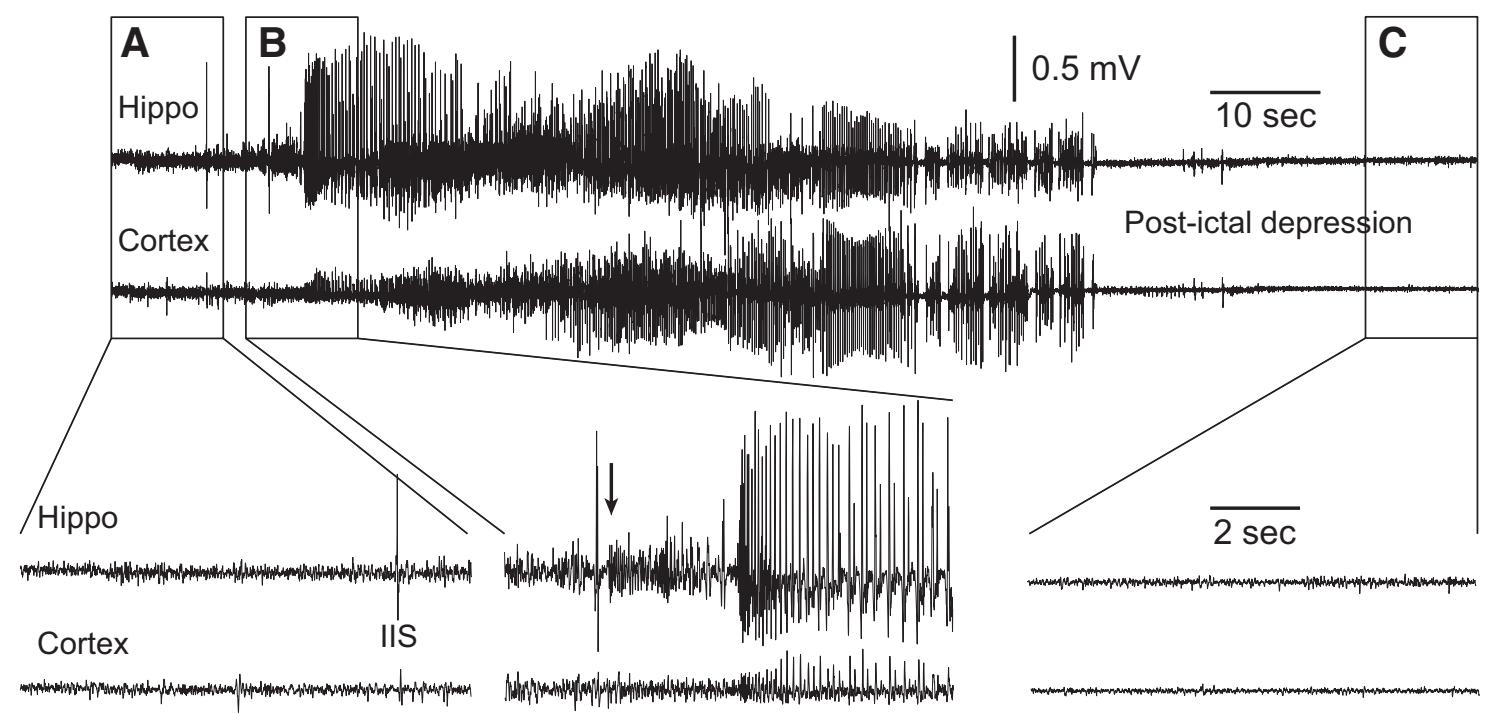

Figure 2. Electrographic activity between and during convulsive seizures. A, Pre-ictal EEG recorded from hippocampus and cortex, with example of an IIS. B, Seizures consisted of high-frequency spikes typically starting in the hippocampus (arrow) and spreading to the cortex, with a gradual slowing of frequency. C, Seizures terminated with post-ictal depression.

other groups including controls. Of the four $\mathrm{St}+\mathrm{TB}$ rats with epilepsy, the seizures typically occurred in clusters of 2-3 (Fig. $4 A$, red lines) at a rate of $7.3 \pm 0.5$ per month. Video recording indicated the convulsions could be severe (stage $4-5$ ). These animals also displayed IISs at a rate of $41 \pm 13.6$ per day $(p<0.001$ compared with controls), grouped into 1-2 d epochs following each seizure cluster (Fig. $4 A$, blue lines). Although the remaining five $\mathrm{St}+\mathrm{TB}$ rats did not present seizures, they produced very frequent IISs, at a rate of $82 \pm 21$ per day (Fig. $4 B ; p<0.001$ compared with controls). The IISs in both groups of rats had similar waveforms (Fig. 4, right traces) and temporal evolution over weeks. No IISs or seizures were recorded in control rats, and very few IISs were detected in the $\mathrm{St}+\mathrm{Sa}$ or noSt $+\mathrm{TB}$ rats (Fig. $4 C$ ), both significantly less than IISs in the St + TB group (Fig. 4D; $p<0.001)$ and not significantly different from controls $(p=0.88$ and 0.83 , respectively). ANOVA indicated significant main effects for both stress and terbutaline on seizure rates $\left(F_{(1,32)}=\right.$ $6.28, p=0.018$ for both $)$ and on IIS rates $\left(F_{(1,32)}=19.46\right.$ and 20.34 , respectively, $p<0.001$ for both). Additionally, there was a significant interaction between the factors of stress and terbutaline for both seizures $\left(F_{(1,32)}=6.23, p=0.018\right)$ and IISs $\left(F_{(1,32)}=\right.$ $17.62, p<0.001)$, indicating that, in contrast to behavioral results, the combination of stress and terbutaline produced enhanced epileptic excitability.

\section{Hippocampal gliosis}

The pattern of GFAP reactivity in hippocampus indicated significant group differences $\left(F_{(3,64)}=21.45, p<0.001\right)$ in astrogliosis that followed both the behavioral and epilepsy results (Fig. 5). Astrogliosis for $\mathrm{St}+\mathrm{TB}$ was the highest, and significantly greater than all other groups $(p \leq 0.01)$. With introduction of stress or terbutaline alone $(\mathrm{St}+\mathrm{Sa}$ and noSt $+\mathrm{TB})$, GFAP immunolabeling increased relative to controls ( $p=0.018$ and 0.022 , respectively), but did not differ from each other $(p=0.94)$. The combination of stress and terbutaline significantly increased GFAP immunoreactivity compared with controls $(p<0.001)$ and terbutaline alone (noSt $+\mathrm{TB} ; p<0.001)$. Main effects for both stress and terbutaline were significant $\left(F_{(1,64)}=29.73\right.$ and 28.55, respectively, $p<0.001$ for both). There was also a significant interaction between these factors $\left(F_{(1,64)}=4.44, p=0.039\right)$, indicating that, similar to epilepsy, the combination of stress and terbutaline produced enhanced hippocampal GFAP reactivity.

\section{Summary of results}

The key results from these experiments are as follows: (1) Either maternal stress or terbutaline, two known human environmental risk factors, cause ASD-like behavior as reflected in the composite ASD score, but although combining of these teratogens does not result in significantly greater behavioral impairment than either alone, a combination is required to cause the dual pathology of autism and epilepsy. (2) Resulting convulsive seizures appear to be initiated in the hippocampus and then spread to the cortex, suggesting that they represent a model of complex partial seizures with secondary generalization, typical of many forms of acquired epilepsy. (3) Rats receiving both maternal stress and terbutaline develop IISs over days and weeks, suggesting a slow evolution and fluctuation of epileptic excitability in both seizure and nonseizure animals. (4) Hippocampal labeling of GFAP (astrocytosis) mirrors the effects of single and combined teratogens on epilepsy, suggesting a potential common mechanism for (or effect of) both autism/epilepsy in this model.

\section{Discussion}

\section{Animal models of ASD-like behavior}

The spectrum of autism is both wide and heterogeneous, presenting a challenge not only for diagnosis (Happé, 2011), but also for creating relevant tests of ASD-like behavior in animal models (Silverman et al., 2010; Patterson, 2011). The initial diagnostic criteria for ASD in humans as defined by the American Psychiatric Association's DSM-IV (American Psychiatric Association, 1994) included three core behavioral symptoms: (1) deficits in social interaction, (2) deficits in communication, and (3) stereotyped/repetitive behavior. Revisions in the more recently published American Psychiatric Association's DSM-5 (American Psychiatric Association, 2013) merged the first two criteria into a more general social-communication factor. Yet, as noted in a recent review of autism-relevant behavioral phenotypes in rats and mice (Servadio et al., 2015), social communication and sociability are still best tested separately, leaving three behavioral tests to assess two core behavioral criteria. Accordingly, we used a 
A
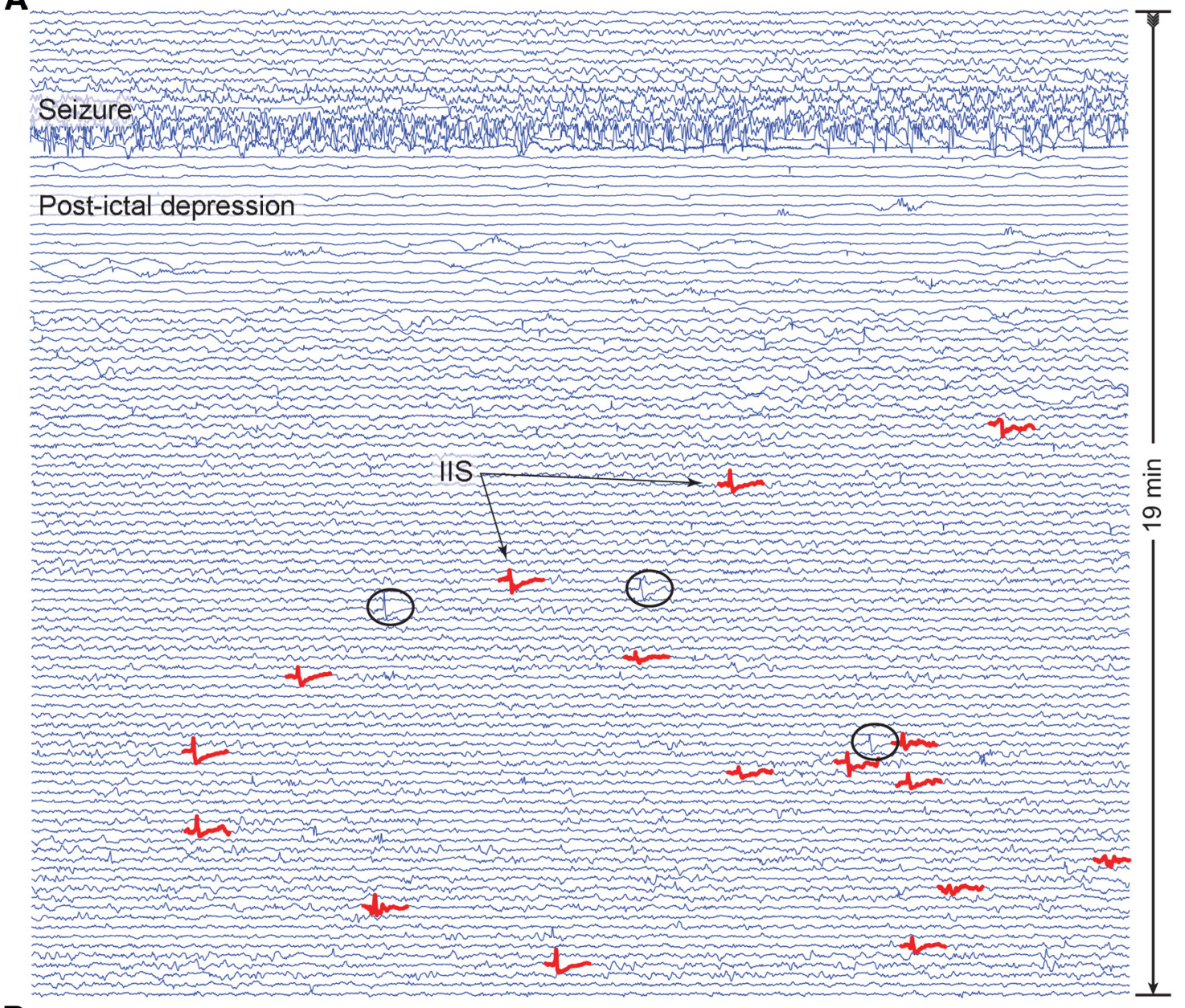

B

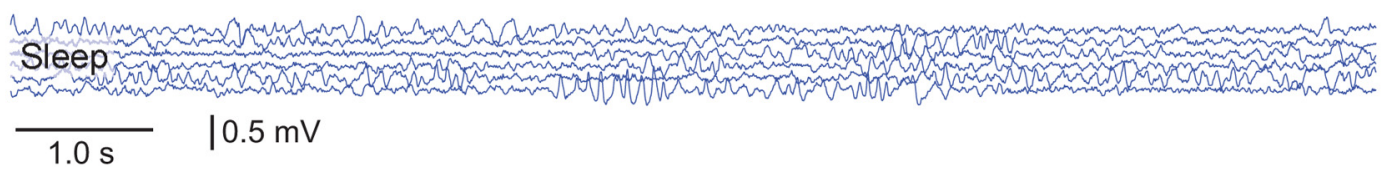

Figure 3. Supervised pattern recognition of IISs. A, Hippocampal EEG is plotted in raster form as $10 \mathrm{~s}$ traces. The threshold for IIS detection (red) was set sufficiently high to avoid missidentification of seizure discharge and slow-wave sleep $(\boldsymbol{B})$. This threshold resulted in $\sim 10 \%$ of IISS (ovals) that went undetected.

social exploration test to measure deficits in social interaction because it is also highly sensitive to anxiety (File and Seth, 2003), a common observation in autism. Ultrasonic vocalizations are the principal form of communication in rats, used among other things to communicate distress (ie, when pups are separated from their dam or surprised by sudden noises), although more needs to be done to understand their variety and social functions (Brudzynski, 2009). Finally, stereotyped/repetitive behavior was assessed by quantifying abnormally long and numerous bouts of self-grooming. Although these tests can only approximate autistic behavior as translated to the rodent, they are similar to tests used by many other laboratories and permit comparison between studies of both environmental (Patterson, 2011) and genetic (Silverman et al., 2010) models of ASD in rats and mice. Future studies using the present model should note that measurements of social exploration and grooming, as well as measurements of epilepsy, were performed during periods including puberty in our rats. Although this is unlikely to have had an influence on epilepsy (Logsdon-Pokorny, 2000), it could influence behavioral measures (Sisk and Zehr, 2005).

\section{Combined prenatal stress and terbutaline are required to produce combined ASD-like behavior and epilepsy}

In agreement with our results, it has been shown by others that prenatal stress (Kinney et al., 2008) or terbutaline (Zerrate et al., 2007) alone produce ASD-like behaviors in rats. Prenatal stress also decreases thresholds for induced seizures (Edwards et al., 2002; Sadaghiani and Saboory, 2010; Qulu et al., 2012). Yet, given the high comorbidity of autism and epilepsy in humans, it is surprising that no single teratogen has been identified that produces both ASD-like behavior and SRS in rats. One possible explanation for this failure could be that it often requires a combination of genetic predisposition and a given teratogen to produce the severe effects reported here (Kim and Leventhal, 2015). However, our data suggest otherwise. Even without genetic predisposition, the combined effects of two environmental teratogens are both sufficient and appear necessary to produce autism/epilepsy.

There has been controversy about the risk of terbutaline, independent of other factors, for human fetal development disorders (Witter et al., 2009; Rodier et al., 2011; Witter, 


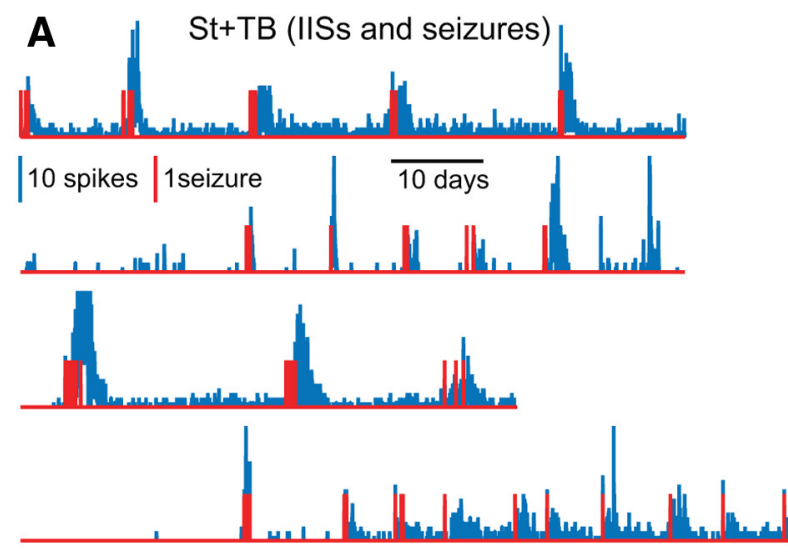

\section{Average IISs}
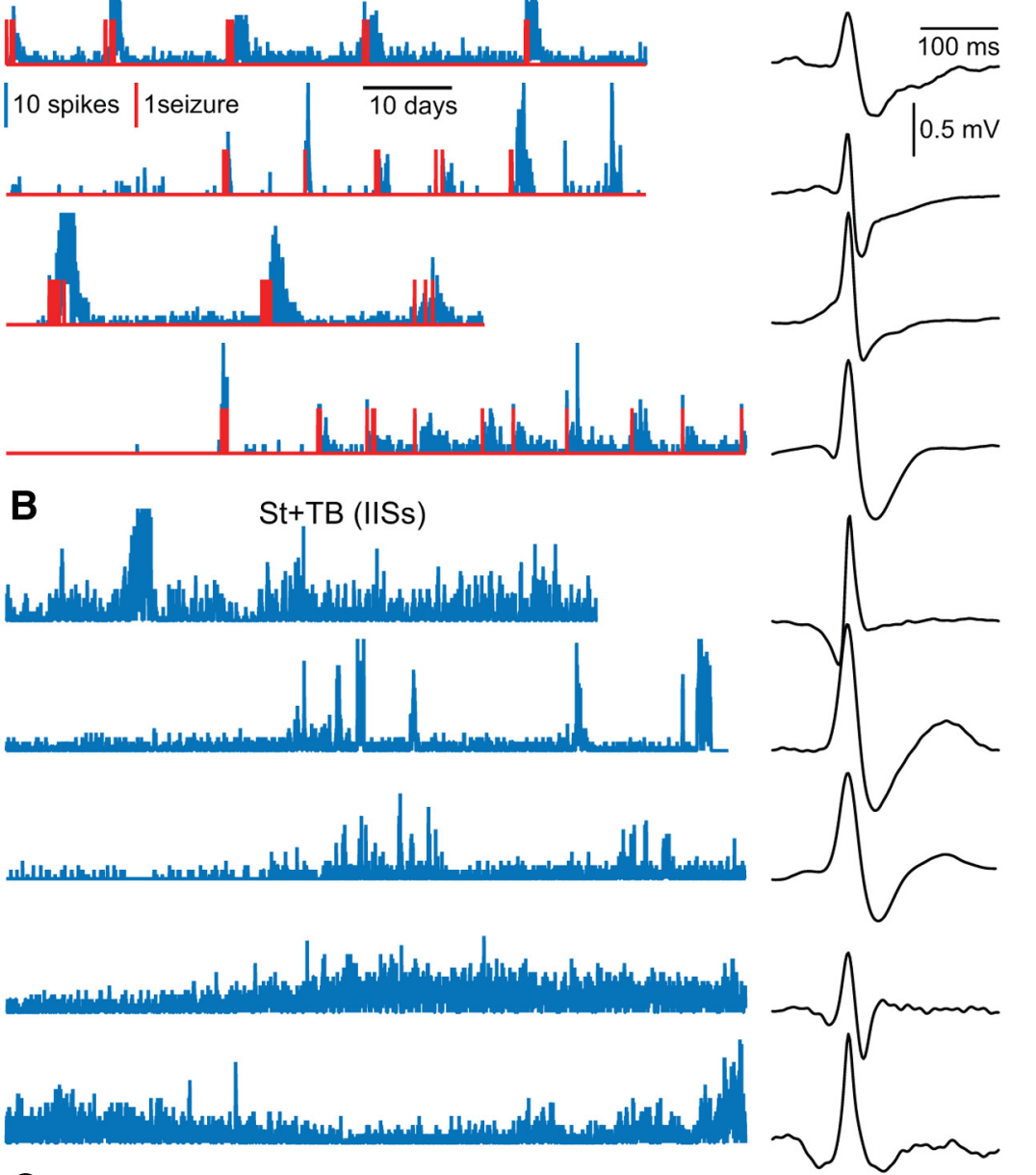

C

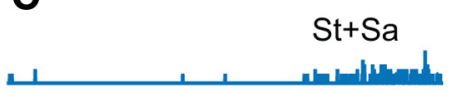

noSt+TB
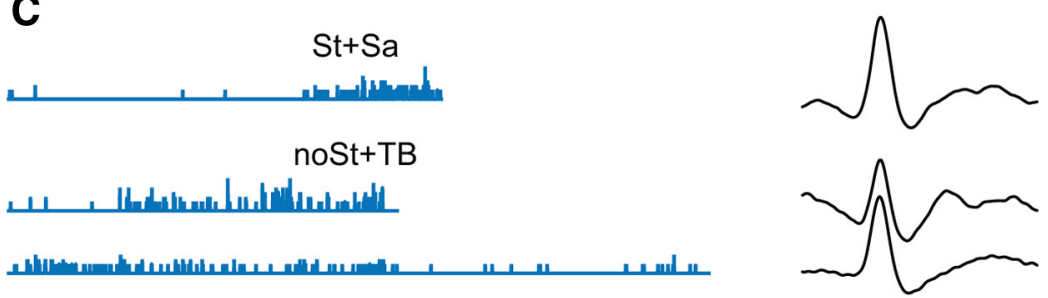

D

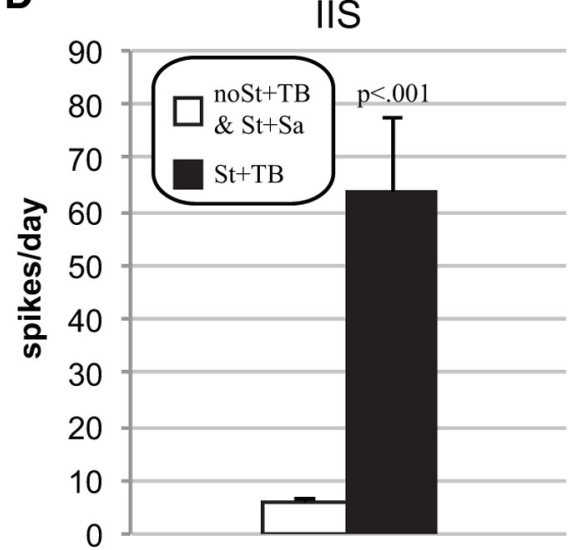

2011). This controversy is based in part on difficulties encountered when inferring human risk from animal models. Additionally, the risk of preterm birth on mortality and developmental outcome may outweigh possible risks introduced by terbutaline. Yet, our data indicate that the true risk of teratogens may be underestimated when studied alone, and that combined effects, in this case terbutaline combined with maternal stress, may be far more severe than either teratogen alone. Thus, although the relative risk of terbutaline use alone compared with that of preterm birth may be low, the present results suggest that realistic risk assessment for human use must account for possible combinatorial effects.

\section{SRSs in the rat model approximate complex partial seizures in ASD patients}

We found that combined maternal stress and terbutaline not only produces severe behavioral deficits, but nonconvulsive epileptiform discharges and convulsive seizures. Although a variety of seizure types have been associated with comorbid autism and epilepsy (Tuchman et al., 1991), complex partial (recently termed "dyscognitive focal"; Berg et al., 2010) seizures are common, reportedly as high as $62-82 \%$ (Gillberg, 1991; Rossi et al., 1995; Steffenburg and Gillberg, 2003). Many of the seizures in acquired human epilepsy, similar to the kainate and pilocarpine models in rats, are convulsive, similar to those recorded here. These seizures are thought to originate in the hippocampus and/or surrounding limbic structures. Electrographically, they generally increase in intensity over many seconds at onset, undergo an evolution in activity over time during the seizure, and show a post-ictal suppression of EEG activity. These characteristics closely resemble the seizures we recorded in all of our epileptic rats. Thus, the seizures produced after the combination of prenatal maternal stress and terbutaline in 2- to 5 -d-old rat pups appear to be a reasonably accurate model of the behavioral, anatomic, and electrographic features of comorbid autism and epilepsy in humans.

\section{$\leftarrow$}

displayed few IISs with little evolution over time and no seizures. D, IIS rates for St + TB rats are higher than combined noST + TB and St + Sa rats. No seizures or IISs were recorded in controls.
Figure 4. Comparison of seizures and IISS. A, IIS counts (blue) typically increased over the first month of recording. Seizures (red) in St $+\mathrm{TB}$ rats came in clusters followed by several day increases in IISS. Averaged IISs detected for each rat are displayed to the right. $\boldsymbol{B}$, Non-ictal St + TB rats also showed a progression of IISS, often with similar phasic increases. $C$, noST + TB and St + Sa rats 


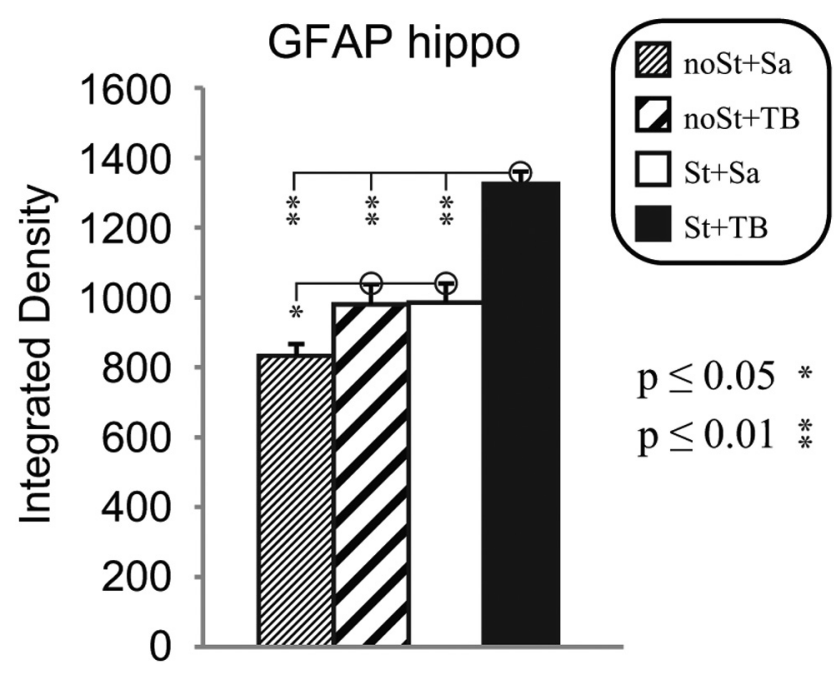

Figure 5. GFAP immunolabeling in hippocampus. GFAP labeling was significantly higher in the ST+ TB group, compared with all other groups. However, both St + Sa and noST + TB were also higher than the noST $+\mathrm{Sa}$ controls.

\section{Nonconvulsive epileptiform discharges in the rat model are common in ASD patients}

We found that non-ictal epileptiform discharges are also prevalent in behaviorally impaired animals without seizures. This finding is in close agreement with observations of nonconvulsive spikes in a larger population of ASD patients than those diagnosed with epilepsy (Ghacibeh and Fields, 2015). When epileptiform EEG abnormalities, in the absence of convulsive seizures, are included in the definition of epilepsy, comorbidity rates rise to as high as 60\% (Lewine et al., 1999; Kim et al., 2006) and have raised questions about whether these discharges may actually have a causal association with ASD (ie, the spikes drive the behavior; Spence and Schneider, 2009). The fact that we observe ASD-like behavior in rats receiving stress or terbutaline alone argues against the likelihood that IISs are required for behavioral effects in this model. It seems more likely that combined stress and terbutaline results in greater limbic impairment (ie, possibly increased hippocampal hyperexcitability), resulting in the parallel development of IISs and behavioral dysfunction. However, the slow (multi-day) increase in IISs repeatedly following seizures, and slow fluctuations of IISs in rats without seizures, suggests that excitability changes indexed by continuous EEG monitoring should provide a model for future work to correlate behavioral changes with IIS prevalence. This finding also encourages closer monitoring of "subclinical" discharges in both ASD and those at risk for epilepsy, to explore their role, if any, in the autism phenotype. In this way, electrophysiology may provide an objective biomarker to complement the current definition of ASD based on core behavioral abnormalities alone. Our data also indicate that ASD-like behavior, at least as quantified by our composite ASD behavior score, may not fully reflect the extent of neurodevelopmental disorder produced by combined teratogens. The combination of stress and terbutaline did not significantly increase ASD-like behavior, whereas this combination made all of the difference in electrophysiological outcome.

\section{Hippocampal inflammation as a possible common mechanism in autism/epilepsy}

Finally, we found that both maternal stress and terbutaline result in increased expression of hippocampal GFAP compared with controls, but that their combination produces significantly more expression, mirroring the epileptic outcomes. This suggests marked hippocampal gliosis in our experimental rats and is in line with the established association between seizures of temporal lobe origin in humans, and hippocampal sclerosis and related astrogliosis (Briellmann et al., 2002). In contrast, there is only sparse evidence for similar hippocampal gliosis in cases of human ASD, possibly due to the small number of autopsy studies that have been conducted on the autistic brain in general and specifically in patients with comorbid autism and epilepsy (DeLong and Heinz, 1997; Tuchman and Rapin, 2002). Hippocampal astrogliosis may reflect increased neuroinflammation, although specific labeling of proinflammatory cytokines would be required to confirm this hypothesis. We have previously shown that neuroinflammation can produce seizures (Rodgers et al., 2009), but it is also recognized that seizures themselves produce neuroinflammation (Vezzani, 2014), potentially forming a positive feedback loop that is both epileptogenic (contributing to the development of chronic epilepsy) and ictogenic (contributing to seizure initiation), and leaving in question which event is causal. However, the fact that our rats with either maternal stress or terbutaline alone and displaying ASD-like behavior, but without epilepsy, also revealed significant astrogliosis compared with controls, suggests that if neuroinflammation is involved, it is precedent and may drive increased excitability, eventually resulting in nonconvulsive spikes as well as seizures.

\section{Conclusions}

We conclude that the maternal environment alone, particularly when multiple risk factors are combined, can impact the development of comorbid autism and epilepsy, and that a common mechanism underlying these disorders may be astrogliosis and neuroinflammation. It is unlikely that the combinatorial effects seen here are restricted to maternal stress and terbutaline. Other maternal environmental risk factors for ASD, such as viral or bacterial infection, are known to have marked neuroimmune consequences and to result in ASD-like behavior in rat models (Patterson, 2009). Our results should therefore inform future human and animal developmental studies of ASD and epilepsy, where teratogens are rarely studied in combination. To our knowledge, this is the first animal model of comorbid autism and epilepsy based exclusively on maternal environmental influences. This model now permits the experimental investigation of the cellular mechanisms and potential intervention strategies for this debilitating comorbid syndrome.

\section{References}

American Psychiatric Association (1994) Diagnostic and statistical manual of mental disorders, Ed 4. Washington DC: American Psychiatric Association.

American Psychiatric Association (2013) Diagnostic and statistical manual of mental disorders, Ed 5. Arlington, VA: American Psychiatric Association.

Avishai-Eliner S, Brunson KL, Sandman CA, Baram TZ (2002) Stressedout, or in (utero)? Trends Neurosci 25:518-524. CrossRef Medline

Berg AT, Berkovic SF, Brodie MJ, Buchhalter J, Cross JH, van Emde Boas W, Engel J, French J, Glauser TA, Mathern GW, Moshé SL, Nordli D, Plouin P, Scheffer IE (2010) Revised terminology and concepts for organization of seizures and epilepsies: report of the ILAE commission on classification and terminology, 2005-2009. Epilepsia 51:676-685. CrossRef Medline

Briellmann RS, Kalnins RM, Berkovic SF, Jackson GD (2002) Hippocampal pathology in refractory temporal lobe epilepsy T2-weighted signal change reflects dentate gliosis. Neurology 58:265-271. CrossRef Medline

Bronson SL, Bale TL (2014) Prenatal stress-induced increases in placental inflammation and offspring hyperactivity are male-specific and amelio- 
rated by maternal anti-inflammatory treatment. Endocrinology 155 : 2635-2646. CrossRef Medline

Brudzynski SM (2009) Communication of adult rats by ultrasonic vocalization: biological, sociobiological, and neuroscience approaches. ILAR J 50:43-50. CrossRef Medline

Charil A, Laplante DP, Vaillancourt C, King S (2010) Prenatal stress and brain development. Brain Res Rev 65:56-79. CrossRef Medline

Christianson JP, Jennings JH, Ragole T, Flyer JG, Benison AM, Barth DS, Watkins LR, Maier SF (2011) Safety signals mitigate the consequences of uncontrollable stress via a circuit involving the sensory insular cortex and bed nucleus of the stria terminalis. Biol Psychiatry 70:458-464. CrossRef Medline

Clancy B, Darlington RB, Finlay BL (2001) Translating developmental time across mammalian species. Neuroscience 105:7-17. CrossRef Medline

Clarke DF, Roberts W, Daraksan M, Dupuis A, McCabe J, Wood H, Snead OC 3rd, Weiss SK (2005) The prevalence of autistic spectrum disorder in children surveyed in a tertiary care epilepsy clinic. Epilepsia 46: 1970-1977. CrossRef Medline

DeLong GR, Heinz ER (1997) The clinical syndrome of early-life bilateral hippocampal sclerosis. Ann Neurol 42:11-17. CrossRef Medline

Dole N, Savitz DA, Hertz-Picciotto I, Siega-Riz AM, McMahon MJ, Buekens P (2003) Maternal stress and preterm birth. Am J Epidemiol 157:14-24. CrossRef Medline

Edwards HE, Dortok D, Tam J, Won D, Burnham WM (2002) Prenatal stress alters seizure thresholds and the development of kindled seizures in infant and adult rats. Horm Behav 42:437-447. CrossRef Medline

El-Kordi A, Winkler D, Hammerschmidt K, Kästner A, Krueger D, Ronnenberg A, Ritter C, Jatho J, Radyushkin K, Bourgeron T, Fischer J, Brose N, Ehrenreich H (2013) Development of an autism severity score for mice using Nlgn4 null mutants as a construct-valid model of heritable monogenic autism. Behav Brain Res 251:41-49. CrossRef Medline

File SE, Seth P (2003) A review of 25 years of the social interaction test. Eur J Pharmacol 463:35-53. CrossRef Medline

Ghacibeh GA, Fields C (2015) Interictal epileptiform activity and autism. Epilepsy Behav 47:158-162. CrossRef Medline

Gillberg C (1991) The treatment of epilepsy in autism. J Autism Dev Disord 21:61-77. CrossRef Medline

Happé F (2011) Criteria, categories, and continua: autism and related disorders in DSM-5. J Am Acad Child Adolesc Psychiatry 50:540-542. CrossRef Medline

Kim HL, Donnelly JH, Tournay AE, Book TM, Filipek P (2006) Absence of seizures despite high prevalence of epileptiform EEG abnormalities in children with autism monitored in a tertiary care center. Epilepsia 47: 394-398. CrossRef Medline

Kim YS, Leventhal BL (2015) Genetic epidemiology and insights into interactive genetic and environmental effects in autism spectrum disorders. Biol Psychiatry 77:66-74. CrossRef Medline

Kinney DK, Munir KM, Crowley DJ, Miller AM (2008) Prenatal stress and risk for autism. Neurosci Biobehav Rev 32:1519-1532. CrossRef Medline

Lam F, Elliott J, Jones JS, Katz M, Knuppel RA, Morrison J, Newman R, Phelan J, Willcourt R (1998) Clinical issues surrounding the use of terbutaline sulfate for preterm labor. Obstet Gynecol Surv 53:S85-S95. CrossRef Medline

Lewine JD, Andrews R, Chez M, Patil AA, Devinsky O, Smith M, Kanner A, Davis JT, Funke M, Jones G, Chong B, Provencal S, Weisend M, Lee RR, Orrison WW Jr (1999) Magnetoencephalographic patterns of epileptiform activity in children with regressive autism spectrum disorders. Pediatrics 104:405-418. CrossRef Medline

Logsdon-Pokorny VK (2000) Epilepsy in adolescents: hormonal considerations. J Pediatr Adolesc Gynecol 13:9-13. CrossRef Medline

Orrù G, Pettersson-Yeo W, Marquand AF, Sartori G, Mechelli A (2012) Using support vector machine to identify imaging biomarkers of neurological and psychiatric disease: a critical review. Neurosci Biobehav Rev 36:1140-1152. CrossRef Medline

Patterson PH (2009) Immune involvement in schizophrenia and autism: etiology, pathology and animal models. Behav Brain Res 204:313-321. CrossRef Medline

Patterson PH (2011) Modeling autistic features in animals. Pediatr Res 69: 34R-40R. CrossRef Medline

Perna R, Loughan A, Perkey H, Tyson K, Perna R, Loughan A, Perkey H, Tyson K (2014) Terbutaline and associated risks for neurodevelopmental disorders. Child Dev Res 2014:e358608.
Qulu L, Daniels WM, Mabandla MV (2012) Exposure to prenatal stress enhances the development of seizures in young rats. Metab Brain Dis 27:399-404. CrossRef Medline

Racine RJ (1972) Modification of seizure activity by electrical stimulation: II. Motor seizure. Electroencephalogr Clin Neurophysiol 32:281-294. CrossRef Medline

Rhodes MC, Seidler FJ, Abdel-Rahman A, Tate CA, Nyska A, Rincavage HL, Slotkin TA (2004) Terbutaline is a developmental neurotoxicant: effects on neuroproteins and morphology in cerebellum, hippocampus, and somatosensory cortex. J Pharmacol Exp Ther 308:529-537. CrossRef Medline

Rice D, Barone S Jr (2000) Critical periods of vulnerability for the developing nervous system: evidence from humans and animal models. Environ Health Perspect 108:511-533. CrossRef Medline

Rodgers KM, Hutchinson MR, Northcutt A, Maier SF, Watkins LR, Barth DS (2009) The cortical innate immune response increases local neuronal excitability leading to seizures. Brain 132:2478-2486. CrossRef Medline

Rodier PM (1988) Structural-functional relationships in experimentally induced brain damage. Prog Brain Res 73:335-348. CrossRef Medline

Rodier P, Miller RK, Brent RL (2011) Does treatment of premature labor with terbutaline increase the risk of autism spectrum disorders? Am J Obstet Gynecol 204:91-94. CrossRef Medline

Rossi PG, Parmeggiani A, Bach V, Santucci M, Visconti P (1995) EEG features and epilepsy in patients with autism. Brain Dev 17:169-174. CrossRef Medline

Sadaghiani MM, Saboory E (2010) Prenatal stress potentiates pilocarpineinduced epileptic behaviors in infant rats both time and sex dependently. Epilepsy Behav 18:166-170. CrossRef Medline

Servadio M, Vanderschuren LJ, Trezza V (2015) Modeling autism-relevant behavioral phenotypes in rats and mice: do "autistic" rodents exist? Behav Pharmacol 26:522-540. CrossRef Medline

Silverman JL, Yang M, Lord C, Crawley JN (2010) Behavioural phenotyping assays for mouse models of autism. Nat Rev Neurosci 11:490-502. CrossRef Medline

Sisk CL, Zehr JL (2005) Pubertal hormones organize the adolescent brain and behavior. Front Neuroendocrinol 26:163-174. CrossRef Medline

Slotkin TA, Seidler FJ (2013) Terbutaline impairs the development of peripheral noradrenergic projections: potential implications for autism spectrum disorders and pharmacotherapy of preterm labor. Neurotoxicol Teratol 36:91-96. CrossRef Medline

Spence SJ, Schneider MT (2009) The role of epilepsy and epileptiform EEGs in autism spectrum disorders. Pediatr Res 65:599-606. CrossRef Medline

Steffenburg S, Steffenburg U, Gillberg C (2003) Autism spectrum disorders in children with active epilepsy and learning disability: comorbidity, preand perinatal background, and seizure characteristics. Dev Med Child Neurol 45:724-730. CrossRef Medline

Tegnér K, Nilsson HT, Persson CG, Persson K, Ryrfeldt A (1984) Elimination pathways of terbutaline. Eur J Respir Dis Suppl 134:93-100. Medline

Tuchman R, Rapin I (2002) Epilepsy in autism. Lancet Neurol 1:352-358. CrossRef Medline

Tuchman RF, Rapin I, Shinnar S (1991) Autistic and dysphasic children. II: Epilepsy. Pediatrics 88:1219-1225. Medline

Tuchman R, Cuccaro M, Alessandri M (2010) Autism and epilepsy: historical perspective. Brain Dev 32:709-718. CrossRef Medline

Vargas DL, Nascimbene C, Krishnan C, Zimmerman AW, Pardo CA (2005) Neuroglial activation and neuroinflammation in the brain of patients with autism. Ann Neurol 57:67-81. CrossRef Medline

Vezzani A (2014) Epilepsy and inflammation in the brain: overview and pathophysiology. Epilepsy Curr 14:3-7. CrossRef Medline

Weinstock M (2008) The long-term behavioural consequences of prenatal stress. Neurosci Biobehav Rev 32:1073-1086. CrossRef Medline

Witter FR (2011) Does treatment of premature labor with terbutaline increase the risk of autism spectrum disorders? Am J Obstet Gynecol 205:e12. CrossRef Medline

Witter FR, Zimmerman AW, Reichmann JP, Connors SL (2009) In utero beta 2 adrenergic agonist exposure and adverse neurophysiologic and behavioral outcomes. Am J Obstet Gynecol 201:553-559. CrossRef Medline

Zerrate MC, Pletnikov M, Connors SL, Vargas DL, Seidler FJ, Zimmerman AW, Slotkin TA, Pardo CA (2007) Neuroinflammation and behavioral abnormalities after neonatal terbutaline treatment in rats: implications for autism. J Pharmacol Exp Ther 322:16-22. CrossRef Medline 